\title{
The "Hyperdense Paraspinal Vein" Sign: A Marker of CSF-Venous Fistula
}

\author{
(DP.G. Kranz, (D).J. Amrhein, (DW.I. Schievink, (DI.O. Karikari, and DL. Gray
}

\begin{abstract}
SUMMARY: CSF-venous fistula is a recently reported cause of spontaneous intracranial hypotension that may occur in the absence of myelographic evidence of CSF leak. Information about this entity is currently very limited, but it is of potential importance given the large percentage of cases of spontaneous intracranial hypotension associated with negative myelography findings. We report 3 additional cases of CSF-venous fistula and describe the "hyperdense paraspinal vein" sign, which may aid in its detection.
\end{abstract}

ABBREVIATION: $\mathrm{SIH}=$ spontaneous intracranial hypotension

S pontaneous intracranial hypotension is thought to result from leakage of CSF from the spinal thecal sac, but in approximately half of cases, no leak is seen on myelography. ${ }^{1,2}$ Patients may be treated with prolonged bed rest or nontargeted lumbar epidural blood patch, but these therapies may not be successful in a substantial proportion of patients. ${ }^{1,3}$ In cases in which targeted treatment is desired (such as when nontargeted lumbar epidural blood patch fails), these cases of SIH with no myelographic evidence of CSF leak can be very challenging, and the inability to determine the site of the leak may preclude effective treatment. It has been previously presumed that these cases may be the result of intermittent or very slow-flow CSF leaks.

Recently, it has been recognized that direct fistulas between CSF and paraspinal veins can be a cause of SIH with negative findings on myelography. In a series of 3 patients, Schievink et $\mathrm{al}^{4}$ reported the presence of such CSF-venous fistulas, diagnosed by using digital subtraction myelography. Two of these cases had negative findings on CT myelography, meaning that they occurred with no concomitant epidural leak of CSF. Presently, it is unclear how frequently this phenomenon occurs and to what extent it may account for some of the cases of SIH in patients with negative findings on myelography. Furthermore, these lesions were identified with digital subtraction myelography, which is not

Received October 28, 2015; accepted after revision December 4.

From the Departments of Radiology (P.G.K., T.J.A., L.G.) and Neurosurgery (I.O.K.), Duke University Medical Center, Durham, North Carolina: and Department of Neurosurgery (W.I.S.), Cedars-Sinai Medical Center, Los Angeles, California.

Please address correspondence to Peter G. Kranz, MD, Department of Radiology, DUMC Box 3808, Duke University Medical Center, Durham, NC 27710; e-mail: peter.kranz@duke.edu; @PeterGKranz

http://dx.doi.org/10.3174/ajnr.A4682 performed at all institutions, is more commonly used in the setting of high-flow CSF leaks, and typically covers only a portion of the total length of the spine. ${ }^{5,6}$ Thus, it would be helpful to identify, from clues on cross-sectional imaging, which patients might benefit from a digital subtraction myelogram and where that imaging should be focused to detect these uncommonly recognized, often subtle lesions.

In this Brief Report, we present 3 additional cases of CSFvenous fistulas in patients with $\mathrm{SIH}$ and describe a novel imaging sign, the "hyperdense paraspinal vein," which may assist in CSFvenous fistula localization.

\section{Cases}

Case 1. A 34-year-old woman had new-onset orthostatic headache and back pain. Brain MR imaging showed dural enhancement, brain sagging, and a venous distention sign. Opening pressure measured $0 \mathrm{~cm} \mathrm{H}_{2} \mathrm{O}$. A blind epidural blood patch improved her symptoms for 2 weeks, but her headaches recurred. CT myelography revealed a focal leak of contrast at the left T8 nerve root (Fig 1). Additionally, a hyperattenuated paraspinal vein was seen at this level, measuring 83-126 HU. The same vein on nonmyelographic CT images measured 27-34 HU. Targeted patching by using blood and fibrin glue was performed at this level but did not result in durable relief. A dynamic myelogram was then obtained, whereby the patient was placed in the left lateral decubitus position with the table tilted feet-down, contrast was injected into the thecal sac, the patient was then slowly tilted head-down, and the contrast passage was monitored by using intermittent fluoroscopy and spot radiographs. This study demonstrated no epidural fluid collection, but there was evidence of a CSF-venous fistula, with myelographic contrast identified in the adjacent paraspinal vein. The patient was taken to the operating room, where a hemi- 

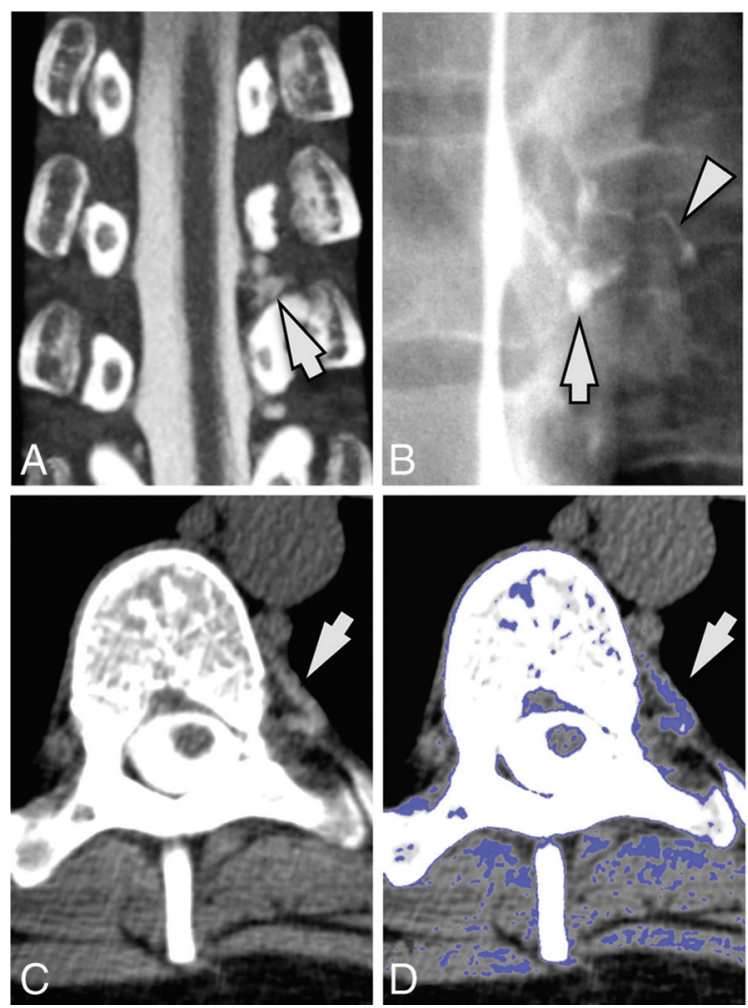

FIG 1. A 34-year-old woman with SIH. A, Coronal image from a CT myelogram shows a low-flow CSF leak inferior to the left T8 nerve root (arrow). B, A subsequent dynamic myelogram obtained with the patient in the left lateral decubitus position shows the area of the leak (arrow) with a fistula to an adjacent paraspinal vein (arrowhead). C, Axial image from her original CT myelogram reveals a hyperattenuated paraspinal vein (arrow). D, Postprocessed image with thresholded color overlay depicting attenuation values from 60 to $140 \mathrm{HU}$ helps improve the conspicuity of this hyperattenuated vein.

laminectomy was performed to expose the nerve root. No free epidural CSF leakage was seen, but an abnormal enlarged vein was seen connecting to a CSF-filled diverticulum of the nerve root, which was coagulated and divided. Following the operation, the positional headache immediately resolved and had not recurred at 4-month follow-up.

Case 2. A 56-year-old man with new-onset positional headaches associated with neck and interscapular pain. Brain MR imaging showed dural enhancement, brain sagging, a venous distention sign, and bilateral subdural collections. Opening pressure measured $3 \mathrm{~cm} \mathrm{H} \mathrm{H}_{2} 0$. Nontargeted lumbar epidural blood patch only helped his symptoms for a few hours. CT myelogram revealed a small focus of CSF leakage adjacent to the right nerve root sleeve at T10-11 (Fig 2). A hyperattenuated paraspinal vein was seen at this level, measuring 93-146 HU. The same vein on nonmyelographic CT images measured 14-32 HU. Targeted patching by using blood and fibrin glue was performed, which only provided 5 days of relief. He re-presented with new subdural hemorrhages and underwent repeat targeted patching 2 additional times during the next month, again without durable relief. He was then taken to the operating room, where a hemilaminectomy was performed to expose the nerve root. No dural defect or CSF leak was seen; however, prominent veins surrounding the nerve root were identified
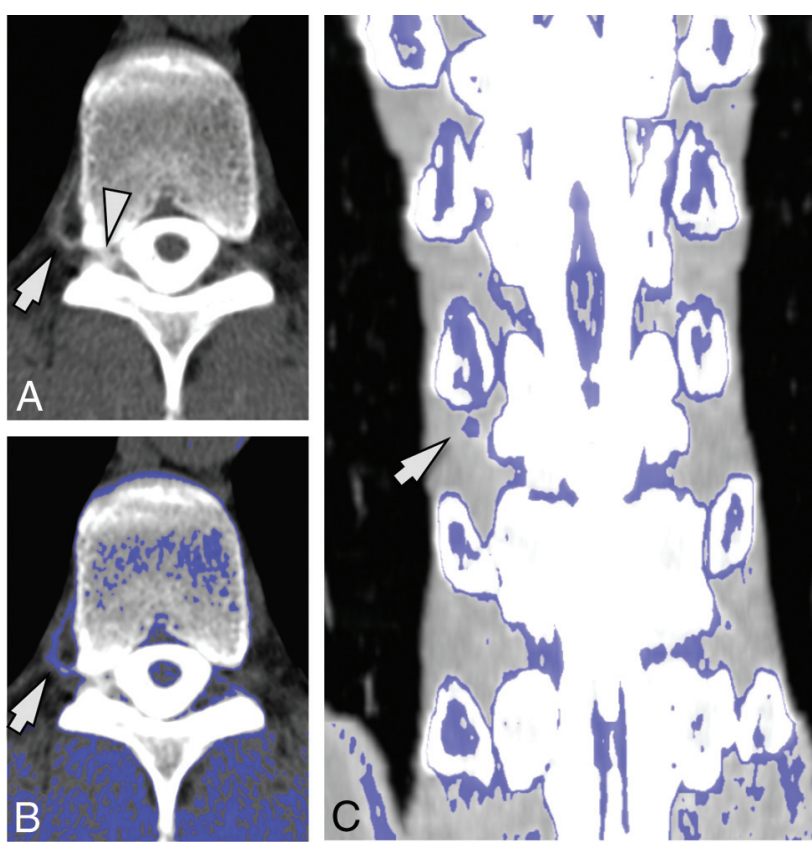

FIG 2. A 56-year-old man with SIH. A, Axial image from a CT myelogram shows a low-flow CSF leak in the right neural foramen at T10-11 (arrowhead). An adjacent hyperattenuated paraspinal vein (arrow) is seen. $B$, Postprocessed image with thresholded color overlay depicting attenuation values from 60 to $140 \mathrm{HU}$ improves the conspicuity of this hyperattenuated vein (arrow). C, Coronal image with thresholded color overlay demonstrates that only the vein at the level and side of the fistula (arrow) shows increased attenuation.

and coagulated. Following surgery, the positional headaches immediately resolved and had not recurred at 2-year follow-up.

Case 3. A 59-year-old woman presented with 5 years of positional headache, neck and bilateral upper extremity pain, and tinnitus. Brain MR imaging showed dural enhancement, brain sagging, and a venous distention sign. Opening pressure measured $10.2 \mathrm{~cm} \mathrm{H}_{2} \mathrm{O}$. A previous blind epidural blood patch did not produce durable relief. CT myelography revealed extensive nerve root sleeve diverticula but no evidence of a CSF leak. A hyperattenuated paraspinal vein was present on the right at T6-7, measuring 85-101 HU (Fig 3). The same vein on nonmyelographic $\mathrm{CT}$ images measured $29 \mathrm{HU}$. She underwent epidural patching by using blood and fibrin glue targeted at multiple nerve root sleeve diverticula on 3 occasions, but her symptoms recurred each time. A digital subtraction myelogram was then obtained, by using the technique previously described, ${ }^{6}$ revealing a CSF-venous fistula on the right at $\mathrm{T} 6-7$. At the operation, a very thin-walled cyst was encountered, to which was attached an extensive venous plexus. The plexus was carefully dissected free and then cauterized. No dural defect was seen intraoperatively. Her positional headaches resolved after surgery and had not recurred at 15-month follow-up.

\section{DISCUSSION}

In this report, we present radiologic and surgical confirmation of the presence of CSF-venous fistulas as an etiology for SIH, first reported in a recent small case series of 3 patients by Schievink et al. ${ }^{4}$ Confirmation of this entity is important in that it identifies an alternative mechanism of CSF volume loss leading to the clinical 

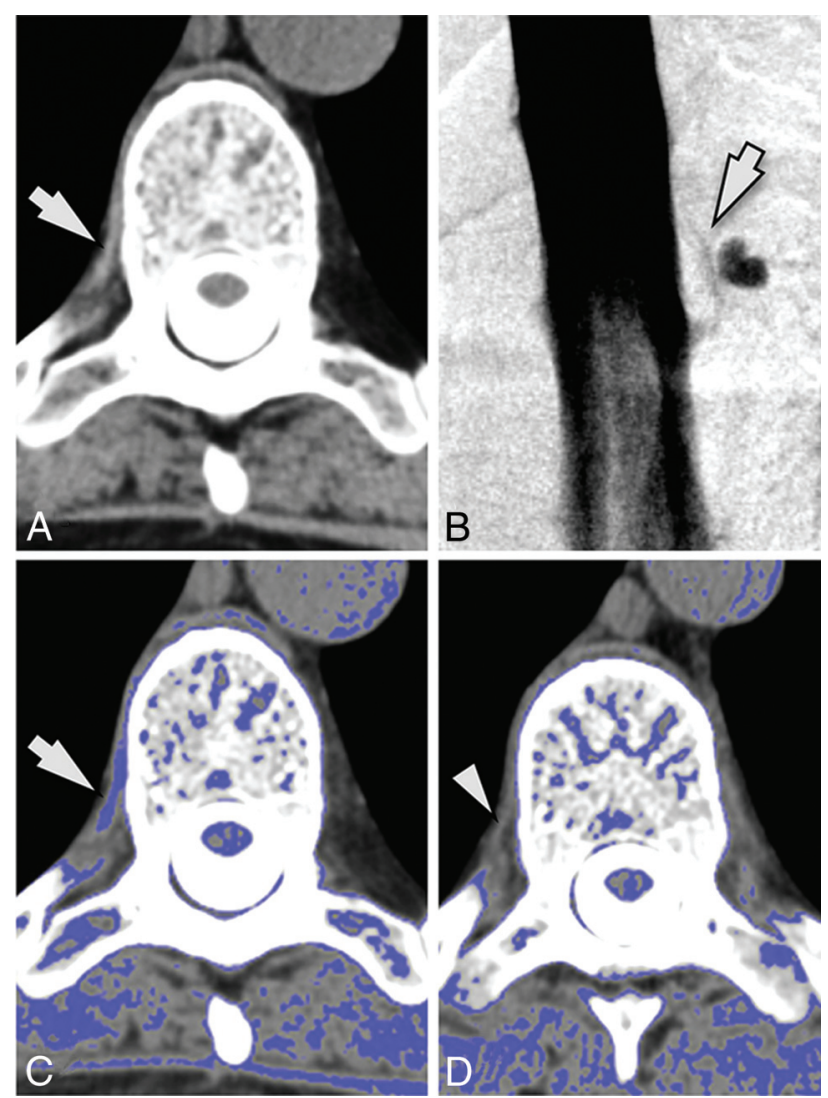

FIG 3. A 59-year-old woman with $\mathrm{SIH}$. A, Axial image from a CT myelogram shows a hyperattenuated paraspinal vein (arrow) at T6-7 on the right. $B$, A subsequent digital subtraction myelogram shows a CSF-venous fistula at this location (arrow). C, Postprocessed image with thresholded color overlay depicting attenuation values from 60 to $140 \mathrm{HU}$ again helps with the identification of this finding (arrow). D, Axial image from an adjacent level (T8-9) where there was no fistula is provided for comparison. Note that the paraspinal vein is not hyperattenuated (arrowhead) and is not identified on the thresholded color overlay.

syndrome of SIH. For the estimated $46 \%-55 \%$ of patients with SIH with no evidence of CSF leakage on myelography, ${ }^{1,2}$ the identification of a fistula may provide the opportunity for targeted treatment. On the basis of our limited experience with CSF-venous fistulas, we have found them to be generally unresponsive to epidural patching, ultimately requiring surgical intervention to obliterate the fistula and its draining veins.

Our series also suggests that CT myelography may provide some clues as to the presence and location of the fistula. In all 3 of our cases, after intrathecal contrast was administered, a hyperattenuated paraspinal vein was identified in close proximity to the site where the CSF fistula was ultimately localized. The average attenuation of the hyperattenuated vessel on postmyelographic CT measured 105.7 $\pm 23.0 \mathrm{HU}$, compared with attenuation values of $27.5 \pm 6.4 \mathrm{HU}$ for the same vessel on nonmyelographic CT (ie, CT performed without intrathecal myelographic contrast).

The conspicuity of this sign was improved by the use of image postprocessing with commercially available software (Aquarius iNtuition, Version 4.4.11; TeraRecon, San Mateo, California).
Image thresholding with color overlay was used to highlight CT attenuation values of $60-140 \mathrm{HU}$; this range was selected empirically on the basis of observed attenuation values in the hyperattenuated-appearing veins compared with normal-appearing paraspinal veins at other levels.

This "hyperdense paraspinal vein" sign presumably represents rapid passage of myelographic contrast into the venous system through the fistula. Under normal conditions, CSF reabsorption into the bloodstream happens at the level of spinal nerve roots, driven by a pressure gradient across arachnoid villi by means of vacuoles that traverse the wall of the villus in a unidirectional manner. ${ }^{7-9}$ CSF-venous fistulas may represent focal rupture or failure of these villi, resulting in persistent, unregulated CSF loss.

Identification of this sign not only suggests that a fistula is present but also aids in its localization. This finding is potentially important because the subtle imaging appearance associated with many of the reported cases of CSF-venous fistula may make them difficult to detect on conventional myelography or digital subtraction myelography unless their presence is suspected, and not all patients with SIH will undergo digital subtraction myelography because its use has previously been generally restricted to a limited number of medical centers where it is typically used for the localization of high-flow CSF leaks. ${ }^{5}$ As future cases of CSFvenous fistulas are identified, it will be useful to determine the sensitivity of this imaging sign, whether there are false-positive causes of hyperattenuated paraspinal veins, whether this entity is ever seen outside of the context of SIH, and whether other imaging techniques or image postprocessing may aid in its detection.

\section{REFERENCES}

1. Sencakova D, Mokri B, McClelland RL. The efficacy of epidural blood patch in spontaneous CSF leaks. Neurology 2001;57:1921-23 CrossRef Medline

2. Luetmer PH, Schwartz KM, Eckel LJ, et al. When should I do dynamic CT myelography? Predicting fast spinal CSF leaks in patients with spontaneous intracranial hypotension. AJNR Am J Neuroradiol 2012;33:690-94 CrossRef Medline

3. Chung SJ, Kim JS, Lee MC. Syndrome of cerebral spinal fluid hypovolemia: clinical and imaging features and outcome. Neurology 2000;55:1321-27 CrossRef Medline

4. Schievink WI, Moser FG, Maya MM. CSF-venous fistula in spontaneous intracranial hypotension. Neurology 2014;83:472-73 CrossRef Medline

5. Hoxworth JM, Trentman TL, Kotsenas AL, et al. The role of digital subtraction myelography in the diagnosis and localization of spontaneous spinal CSF leaks. AJR Am J Roentgenol 2012;199:649-53 CrossRef Medline

6. Hoxworth JM, Patel AC, Bosch EP, et al. Localization of a rapid CSF leak with digital subtraction myelography. AJNR Am J Neuroradiol 2009;30:516-19 CrossRef Medline

7. Levine JE, Povlishock JT, Becker DP. The morphological correlates of primate cerebrospinal fluid absorption. Brain Res 1982;241: 31-41 CrossRef Medline

8. Cutler RW, Page L, Galicich J, et al. Formation and absorption of cerebrospinal fluid in man. Brain 1968;91:707-20 CrossRef Medline

9. Edsbagge M, Tisell M, Jacobsson L, et al. Spinal CSF absorption in healthy individuals. Am J Physiol Regul Integr Comp Physiol 2004; 287:R1450-55 CrossRef Medline 